\title{
Effect of gravity on visco-elastic surface waves in solids involving time rate of strain and stress of first order
}

\author{
TAPAN KUMAR DAS ${ }^{\dagger}$ and P R SENGUPTA* \\ Department of Mathematics, University of Kalyani, PO Kalyani 741235 , \\ Dist. Nadia, India \\ ${ }^{\dagger}$ Present address: Indian Institute of Mechanics of Continua, c/o \\ Dr. Pran Ranjan Sengupta \\ *Present address: 201, Manicktala Main Road, Suite No. 42, Calcutta \\ 700054, India
}

MS received 9 September 1991; revised 7 February 1992

\begin{abstract}
The aim of the present paper is to study the effect of gravity on visco-elastic surface waves in solids. The wave velocity equations are deduced from Biot's theory of initial stress on the assumption that gravity creates a type of initial stress - hydrostatic in nature. Resulting equations are used to investigate surface waves of the Rayleigh, Love and Stoneley types. Results are in good agreement with corresponding classical results when gravity and viscosity are neglected.
\end{abstract}

Keywords. Visco-elastic; first order; surface waves; gravity.

\section{Introduction}

Usefulness of surface waves is well recognised in the study of earthquakes, seismology, geophysics and geodynamics. The theory of surface waves has been widely developed by Rayleigh (1885), Voigt (1887), Stoneley (1924), Ewing et al (1957, pp. 257-259, 311), Hunter (1960, pp. 1-57), Bland (1960, pp. 30-75), Flugge (1967, pp. 3-21) and Jeffreys (1959, pp. 35-38).

Effects of gravity, curvature and viscosity are not considered in detail. Considering the effect of gravity in the problem of propagation of waves in solids, in particular, on an elastic globe has been discussed first by Bromwich (1898). Subsequently, the investigations of the effects of gravity was considered by Love (1911, pp. 144-178) in his text, Some problems of geodynamics, wherein he exhibited that the velocity of Rayleigh waves is increased to a significant extent by the gravitational field when wavelengths are large. Biot (1965, pp. 44-45) investigated the effect of gravity on Rayleigh waves by assuming gravity to create a type of initial stress of hydrostatic nature and the medium to be incompressible. Adapting the same theory of initial stress and using the dynamical equations of motion for a homogeneous isotropic elastic solid medium under the initial stress, some problems of waves and vibrations 
have been studied by different investigators. De \& Sengupta (1973) studied the effects of gravity on elastic waves and vibrations and also on the propagation of waves in an elastic layer (De \& Sengupta 1974). Das \& Sengupta (1990a) considered problems of surface waves in general visco-elastic media of higher order and also surface waves in thermo-visco-elastic media considering time rate of stress and strain of higher order (Das \& Sengupta 1990b). Roy \& Sengupta (1983a) investigated the rotatory vibration of a general visco-elastic solid sphere and also the radial vibration of a general visco-elastic solid sphere (Roy \& Sengupta 1983b). The details are found in the work of Eringen \& Suhubi (1975, pp. 518, 524-530, 622, 830).

In this paper an attempt has been made to formulate the equations of motion in visco-elastic media under the influence of gravity. Starting from the dynamical equations of motion for a homogeneous isotropic elastic solid medium under initial stress, as presented by Biot (1965, pp. 273-281), the authors have derived the wave velocity equations satisfied by displacement potentials $\phi$ and $\psi$ to account for gravity and viscosity. This theory is then applied to the particular examples of Rayleigh waves, Love waves and Stoneley waves. Final wave velocity equations in each case are in good agreement with the corresponding classical results when the gravity field and the viscous field are neglected.

\section{Formulation of the problem}

Consider two homogeneous semi-infinite visco-elastic solid media $M_{1}$ and $M_{2}$ welded in contact in the influence of gravity (figure 1). Suppose that the media are separated by a plane horizontal boundary, extending to infinitely great distance from the origin, $M_{2}$ being above $M_{1}$. As a reference co-ordinate system we consider a set of orthogonal cartesian axes $0 x_{1} x_{2} x_{3}$, the origin 0 being any point on the boundary and $0 x_{3}$ pointing normally to $M_{1}$. Consider the possibility of a type of wave travelling in the positive $x_{1}$-direction in such a manner that the disturbance is largely confined to the neighbourhood of the boundary and at any instant all particles in any line parallel to the $x_{3}$-axis have equal displacements. These two assumptions conclude that the

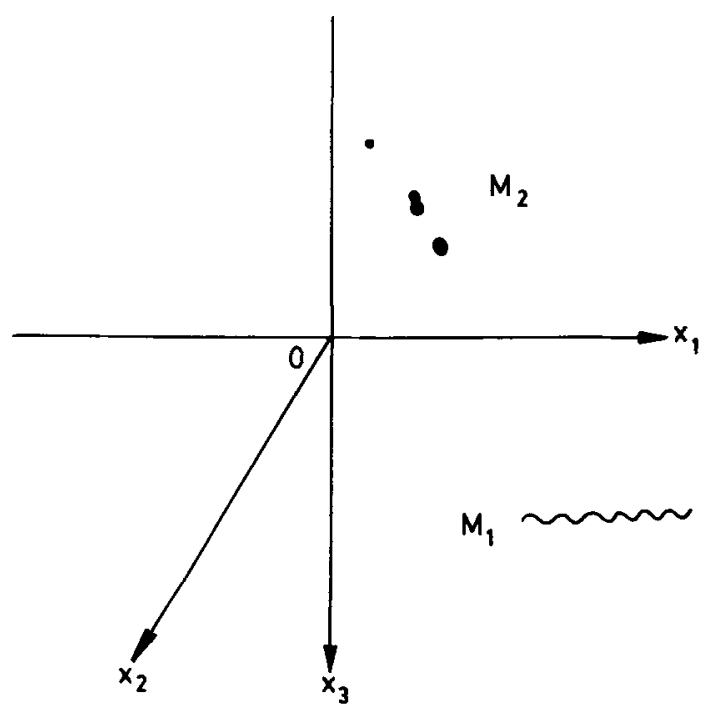

Figure 1. Interface geometry. 
wave is a surface wave and all partial derivatives with respect to $x_{2}$ are zero.

Let us assume that $u_{1}, u_{2}$ and $u_{3}$ are the components of displacement at any point $\left(x_{1}, x_{2}, x_{3}\right)$ at time $t$. We may separate out the purely dilatational and purely rotational disturbances associated with the components $u_{1}$ and $u_{3}$ by introducing two displacement potentials $\phi$ and $\psi$ in the form

$$
u_{1}=\frac{\partial \phi}{\partial x_{1}}-\frac{\partial \psi}{\partial x_{3}}, \quad u_{3}=\frac{\partial \phi}{\partial x_{3}}+\frac{\partial \psi}{\partial x_{1}}
$$

where $\phi$ and $\psi$ are the functions of the co-ordinates $x_{1}, x_{3}$, and $t$ and

$$
\begin{aligned}
& \nabla^{2} \phi=\Delta, \quad \nabla^{2} \psi=\frac{\partial u_{3}}{\partial x_{1}}-\frac{\partial u_{1}}{\partial x_{3}} \\
& \nabla^{2}=\frac{\partial^{2}}{\partial x_{1}^{2}}+\frac{\partial^{2}}{\partial x_{2}^{2}}+\frac{\partial^{2}}{\partial x_{3}^{2}}, \quad \Delta=\frac{\partial u_{1}}{\partial x_{1}}+\frac{\partial u_{2}}{\partial x_{2}}+\frac{\partial u_{3}}{\partial x_{3}} .
\end{aligned}
$$

The component $u_{2}$ is associated with purely distortional movement. We mark that $\phi, \psi$ and $u_{2}$ are respectively associated with $P$-waves, $S V$-waves and $S H$-waves (Bullen 1965, pp. 252-265). The symbols have their usual meanings.

The dynamical equations of motion for the three-dimensional problem under the state of initial stress and gravity (Biot 1965, pp. 44-45, 273-281) are

$$
\begin{aligned}
& \frac{\partial \sigma_{11}}{\partial x_{1}}+\frac{\partial \sigma_{12}}{\partial x_{2}}+\frac{\partial \sigma_{13}}{\partial x_{3}}+\rho g \frac{\partial u_{3}}{\partial x_{1}}=\rho \frac{\partial^{2} u_{1}}{\partial t^{2}}, \\
& \frac{\partial \sigma_{21}}{\partial x_{1}}+\frac{\partial \sigma_{22}}{\partial x_{2}}+\frac{\partial \sigma_{23}}{\partial x_{3}}+\rho g \frac{\partial u_{3}}{\partial x_{2}}=\rho \frac{\partial^{2} u_{2}}{\partial t^{2}}, \\
& \frac{\partial \sigma_{31}}{\partial x_{1}}+\frac{\partial \sigma_{32}}{\partial x_{2}}+\frac{\partial \sigma_{33}}{\partial x_{3}}-\rho g\left(\frac{\partial u_{1}}{\partial x_{1}}+\frac{\partial u_{2}}{\partial x^{2}}\right)=\rho \frac{\partial^{2} u_{3}}{\partial t^{2}},
\end{aligned}
$$

where $\rho$ is the density of the homogeneous medium, $g$ is the acceleration due to gravity and $\sigma_{i j}$ are the stress components.

The stress-strain relation according to Voigt (1887) in an isotropic visco-elastic solid medium of first order is

$$
\left(\eta_{1}+\eta_{2} \frac{\partial}{\partial t}\right) \sigma_{i j}=\left(\lambda_{1}+\lambda_{2} \frac{\partial}{\partial t}\right) \Delta \delta_{i j}+2\left(\mu_{1}+\mu_{2} \frac{\partial}{\partial t}\right) e_{i j},
$$

where $\eta_{1}, \lambda_{1}, \mu_{1}$ are elastic constants with $\eta_{2}, \lambda_{2}$ and $\mu_{2}$ accounting for viscosity, $e_{i j}$ is the strain tensor and $\delta_{i j}$ is the Kronecker symbol.

Substituting (3) in (2), we obtain displacement equations of motion in a first-order visco-elastic medium under the influence of gravity as

$$
\begin{aligned}
& {\left[\left(\lambda_{1}+\mu_{1}\right)+\left(\lambda_{2}+\mu_{2}\right) \frac{\partial}{\partial t}\right] \frac{\partial \Delta}{\partial x_{1}}+\left(\mu_{1}+\mu_{2} \frac{\partial}{\partial t}\right) \nabla^{2} u_{1}+\rho g\left(\eta_{1}+\eta_{2} \frac{\partial}{\partial t}\right) \frac{\partial u_{3}}{\partial x_{1}}} \\
& =\rho\left(\eta_{1}+\eta_{2} \frac{\partial}{\partial t}\right) \frac{\partial^{2} u_{1}}{\partial t^{2}} \\
& \left(\mu_{1}+\mu_{2} \frac{\partial}{\partial t}\right) \nabla^{2} u_{2}=\rho\left(\eta_{1}+\eta_{2} \frac{\partial}{\partial t}\right) \frac{\partial^{2} u_{2}}{\partial t^{2}}
\end{aligned}
$$




$$
\begin{aligned}
{\left[\left(\lambda_{1}+\mu_{1}\right)+\left(\lambda_{2}+\mu_{2}\right) \frac{\partial}{\partial t}\right] } & \frac{\partial \Delta}{\partial x_{3}}+\left(\mu_{1}+\mu_{2} \frac{\partial}{\partial t}\right) \nabla^{2} u_{3} \\
& -\rho g\left(\eta_{1}+\eta_{2} \frac{\partial}{\partial t}\right) \frac{\partial u_{1}}{\partial x_{1}}=\rho\left(\eta_{1}+\eta_{2} \frac{\partial}{\partial t}\right) \frac{\partial^{2} u_{3}}{\partial t^{2}}
\end{aligned}
$$

where $\rho, \eta_{1}, \eta_{2}, \lambda_{1}, \lambda_{2}, \mu_{1}, \mu_{2}$ denote the properties of the medium $M_{1}$ and those with dashes the properties of the medium $M_{2}$.

Introducing (1) into (4), we get the following wave equations in $M_{1}$ satisfied by $\phi$, $\psi$ and $u_{2}$

$$
\begin{aligned}
& \frac{\partial^{2} \phi}{\partial t^{2}}=\left(V_{1 T}^{2}+V_{2 T}^{2} \frac{\partial}{\partial t}\right) \nabla^{2} \phi / L+g \frac{\partial \psi}{\partial x_{1}} \\
& \frac{\partial^{2} \psi}{\partial t^{2}}=\left(V_{1 S}^{2}+V_{2 s}^{2} \frac{\partial}{\partial t}\right) \nabla^{2} \psi / L-g \frac{\partial \phi}{\partial x_{1}} \\
& \frac{\partial^{2} u_{2}}{\partial t^{2}}=\left(V_{1 s}^{2}+V_{2 s}^{2} \frac{\partial}{\partial t}\right) \nabla^{2} u_{2} / L
\end{aligned}
$$

where

$$
\begin{aligned}
& V_{1 T}^{2}=\left(\lambda_{1}+2 \mu_{1}\right) / \rho, \quad V_{2 T}^{2}=\left(\lambda_{2}+2 \mu_{2}\right) / \rho \\
& V_{1 S}^{2}=\mu_{1} / \rho, \quad V_{2 S}^{2}=\mu_{2} / \rho, \quad L=\eta_{1}+\eta_{2} \frac{\partial}{\partial t}
\end{aligned}
$$

and similar relations in $M_{2}$ with $\rho, \eta_{1}, \eta_{2}, \lambda_{1}, \lambda_{2}, \mu_{1}, \mu_{2}$ replaced by $\rho^{1}, \eta_{1}^{\mathbf{i}}, \eta_{2}^{1}, \lambda_{1}^{1}$, $\lambda_{2}^{1}, \mu_{1}^{1}, \mu_{2}^{1}$ and so on.

\subsection{Boundary conditions}

The boundary conditions are:

(i) The component of displacement at the interface between the media $M_{1}$ and $M_{2}$ must be continuous at all points and times.

(ii) The stress components $\sigma_{31}, \sigma_{32}, \sigma_{33}$ must be continuous across the interface, i.e.

$$
\begin{aligned}
& L \sigma_{31}=\left(\mu_{1}+\mu_{2} \frac{\partial}{\partial t}\right)\left(2 \frac{\partial^{2} \phi}{\partial x_{1} \partial x_{3}}+\frac{\partial^{2} \psi}{\partial x_{1}^{2}}-\frac{\partial^{2} \psi}{\partial x_{3}^{2}}\right), \\
& L \sigma_{32}=\left(\mu_{1}+\mu_{2} \frac{\partial}{\partial t}\right) \frac{\partial u_{2}}{\partial x_{3}} \\
& L \sigma_{33}=\left(\lambda_{1}+\lambda_{2} \frac{\partial}{\partial t}\right) \nabla^{2} \phi+2\left(\mu_{1}+\mu_{2} \frac{\partial}{\partial t}\right)\left(\frac{\partial^{2} \phi}{\partial x_{3}^{2}}+\frac{\partial^{2} \psi}{\partial x_{1} \partial x_{3}}\right)
\end{aligned}
$$

and similar expressions for $M_{2}$ across the interface between $M_{1}$ and $M_{2}$ must be continuous at all points and times. 


\section{Solutions of the problem}

Let us take the solutions of (5) in the exponential form

$$
\left(\phi, \psi, u_{2}\right)=\left[\hat{\phi}\left(x_{3}\right), \hat{\psi}\left(x_{3}\right), \hat{u}_{2}\left(x_{3}\right) \exp \left[i\left(\eta x_{1}-\omega t\right)\right]\right.
$$

for the medium $M_{1}$ and similar solutions for $M_{2}$, the functions $\hat{\phi}, \hat{\psi}, \hat{u}_{2}$ being replaced by $\hat{\phi}^{\prime}, \hat{\psi}^{\prime}, \hat{u}_{2}^{1}$.

Putting (7) in (5), we get a set of differential equations for the medium $M_{1}$ as follows

$$
\begin{aligned}
& {\left[\frac{\mathrm{d}^{2}}{\mathrm{~d} x_{3}^{2}}-\left(\eta^{2}-\omega^{2} \eta_{K}^{*} / V_{K T}^{2}\right)\right] \hat{\phi}=-i g \eta \hat{\psi} \times \eta_{K}^{*} / V_{K T}^{2},} \\
& {\left[\frac{\mathrm{d}^{2}}{\mathrm{~d} x_{3}^{2}}-\left(\eta^{2}-\omega^{2} \eta_{K}^{*} / V_{K S}^{2}\right)\right] \hat{\psi}=i g \eta \hat{\phi} \times \eta_{K}^{*} / V_{K S}^{2},} \\
& {\left[\frac{\mathrm{d}^{2}}{\mathrm{~d} x_{3}^{2}}-\left(\eta^{2}-\omega^{2} \eta_{k}^{*} / V_{K S}^{2}\right)\right] \hat{u}_{2}=0,}
\end{aligned}
$$

where

$$
\eta_{K}^{*}=\left(\eta_{1}-i \omega \eta_{2}\right), \quad V_{K T}^{2}=V_{1 T}^{2}-i \omega V_{2 T}^{2}, \quad V_{K S}^{2}=V_{1 S}^{2}-i \omega V_{2 S}^{2} .
$$

Similar relations for $M_{2}$ can be obtained replacing $\hat{\phi}, \hat{\psi}, \hat{u}_{2}, \eta_{1}, \eta_{2}, V_{1 T}, V_{2 T}, V_{1 S}$, $V_{2 S}, \eta_{K}^{*}, V_{K T}, V_{K s}, \lambda_{1}, \mu_{1}, \lambda_{2}, \mu_{2}, \rho$ by dashes.

Clearly, these equations (8) have exponential solutions and in order that $\phi, \psi$ and $u_{2}$ describe surface waves, they must become vanishingly small as $x_{3} \rightarrow \infty$.

Hence for the medium $M_{1}$, we have

$$
\begin{aligned}
& \phi=\left[A_{1} \exp \left[-x_{3}\right]\left(\eta^{2}-\zeta_{1}^{2}\right)^{\frac{1}{2}}+A_{2} \exp \left[-x_{3}\right]\left(\eta^{2}-\zeta_{2}^{2}\right)^{\frac{1}{2}}\right] \exp \left[i\left(\eta x_{1}-\omega t\right)\right] \\
& \psi=\left[B_{1} \exp \left[-x_{3}\right]\left(\eta^{2}-\zeta_{1}^{2}\right)^{\frac{1}{2}}+B_{2} \exp \left[-x_{3}\right]\left(\eta^{2}-\zeta_{2}^{2}\right)^{\frac{1}{2}}\right] \exp \left[i\left(\eta x_{1}-\omega t\right)\right] \\
& u_{2}=\left[C \exp \left[-x_{3}\right]\left(\eta^{2}-\omega^{2} \eta_{k}^{*} / V_{K S}^{2}\right)^{\frac{1}{2}}\right] \exp \left[i\left(\eta x_{1}-\omega t\right)\right] .
\end{aligned}
$$

We have similarly those for the medium $M_{2}$ as

$$
\begin{aligned}
& \phi^{\prime}=\left[A_{1}^{\prime} \exp \left[x_{3}\right]\left(\eta^{2}-\zeta_{1}^{\prime 2}\right)^{\frac{1}{2}}+A_{2}^{\prime} \exp \left[x_{3}\right]\left(\eta^{2}-\zeta_{2}^{\prime 2}\right)^{\frac{1}{2}}\right] \exp \left[i\left(n x_{1}-\omega t\right)\right] \\
& \psi^{\prime}=\left[B_{1}^{\prime} \exp \left[x_{3}\right]\left(\eta^{2}-\zeta_{1}^{\prime 2}\right)^{\frac{1}{2}}+B_{2}^{\prime} \exp \left[x_{3}\right]\left(\eta^{2}-\zeta_{2}^{\prime 2}\right)^{\frac{1}{2}}\right] \exp \left[i\left(\eta x_{1}-\omega t\right)\right] \\
& u_{2}^{\prime}=\left[C^{\prime} \exp \left[x_{3}\right]\left(\eta^{2}-\omega^{2} \eta_{K}^{*} / V_{K S}^{\prime 2}\right)^{\frac{1}{2}}\right] \exp \left[i\left(\eta x_{1}-\omega t\right)\right]
\end{aligned}
$$

where $\zeta_{j}^{2}$ and $\zeta_{j}^{\prime 2}(j=1,2)$ are respectively the roots of the equations

$$
\begin{gathered}
{\left[\omega^{2}-\zeta^{2} \times V_{K S}^{2} / \eta_{K}^{*}\right]\left[\omega^{2}-\zeta^{2} \times V_{K T}^{2} / \eta_{K}^{*}\right]-g^{2} \eta^{2}=0,} \\
{\left[\omega^{2}-\zeta^{\prime 2} \times V_{K S}^{\prime 2} / \eta_{K}^{* \prime}\right]\left[\omega^{2}-\zeta^{\prime 2} \times V_{K T}^{\prime 2} / \eta_{K}^{* \prime}\right]-g^{2} \eta^{2}=0}
\end{gathered}
$$


and

with

$$
B_{1}=\alpha_{1} A_{1}, \quad B_{2}=\alpha_{2} A_{2}, \quad B_{1}^{\prime}=\alpha_{1}^{\prime} A_{1}^{\prime}, \quad B_{2}^{\prime}=\alpha_{2}^{\prime} A_{2}^{\prime},
$$

$$
\alpha_{j}=i g \eta /\left(\omega^{2}-\zeta_{j}^{2} \times V_{K S}^{2} / \eta_{k}^{*}\right), \quad \alpha_{j}^{\prime}=i g \eta /\left(\omega^{2}-\zeta_{j}^{\prime 2} \times V_{K S}^{\prime 2} / \eta_{K}^{* \prime}\right), \quad(j=1,2)
$$

In evaluating

$$
\left(\eta^{2}-\zeta^{2}\right)^{\frac{1}{2}}, \quad\left(\eta^{2}-\zeta^{\prime 2}\right)^{\frac{1}{2}}, \quad\left(\eta^{2}-\omega^{2} \times \eta_{K}^{*} / V_{K S}^{2}\right)^{\frac{1}{2}}, \quad\left(\eta^{2}-\omega^{2} \times \eta_{K}^{* \prime} / V_{K S}^{\prime 2}\right)^{\frac{1}{2}},
$$

the root with positive real part will be taken.

Applying boundary conditions (i) and (ii), we obtain

$$
\begin{aligned}
& {\left[1-i \alpha_{1} Q_{1}\right] A_{1}+\left[1-i \alpha_{2} Q_{2}\right] A_{2}=\left[1+i \alpha_{1}^{\prime} Q_{1}^{\prime}\right] A_{1}^{\prime}+\left[1+i \alpha_{2}^{\prime} Q_{2}^{\prime}\right] A_{2}^{\prime} \text {, }} \\
& C=C^{\prime} \text {, } \\
& {\left[\alpha_{1}+i Q_{1}\right] A_{1}+\left[\alpha_{2}+i Q_{2}\right] A_{2}=\left[\alpha_{1}^{\prime}-i Q_{1}^{\prime}\right] A_{1}^{\prime}+\left[\alpha_{2}^{\prime}-i Q_{2}^{\prime}\right] A_{2}^{\prime} \text {, }} \\
& \rho \times\left(V_{K S}^{2} / \eta_{K}^{*}\right)\left[\left\{2 i Q_{1}+\left(1+Q_{1}^{2}\right) \alpha_{1}\right\} A_{1}+\left\{2 i Q_{2}+\left(1+Q_{2}^{2}\right) \alpha_{2}\right\} A_{2}\right] \\
& =\rho^{\prime} \times\left(V_{K S}^{\prime 2} / \eta_{K}^{*^{\prime}}\right)\left[\left\{-2 i Q_{1}^{\prime}+\left(1+Q_{1}^{\prime 2}\right) \alpha_{1}^{\prime}\right\} A_{1}^{\prime}+\left\{-2 i Q_{2}^{\prime}+\left(1+Q_{2}^{\prime 2}\right) \alpha_{2}^{\prime}\right\} A_{2}^{\prime}\right] \text {, } \\
& -\rho \times\left(V_{K S}^{2} / \eta_{K}^{*}\right)\left(\eta^{2}-\omega^{2} \eta_{K}^{*} / V_{K S}^{2}\right)^{\frac{1}{2}} \times C \\
& =\rho^{\prime} \times\left(V_{K S}^{\prime 2} / \eta_{K}^{* \prime}\right)\left(\eta^{2}-\omega^{2} \eta_{K}^{* \prime} / V_{K S}^{\prime 2}\right)^{\frac{1}{2}} \times C^{\prime}, \\
& \left(\rho / \eta_{K}^{*}\right)\left[\left\{V_{K T}^{2}\left(Q_{1}^{2}-1\right)+2 V_{K S}^{2}\left(1-i \alpha_{1} Q_{1}\right)\right\} A_{1}+\left\{V_{K T}^{2}\left(Q_{2}^{2}-1\right)+\right.\right. \\
& \left.\left.+2 V_{K S}^{2}\left(1-i \alpha_{2} Q_{2}\right)\right\} A_{2}\right] \\
& =\left(\rho^{\prime} / \eta_{K}^{* \prime}\right)\left[\left\{V_{K T}^{\prime 2}\left(Q_{1}^{\prime 2}-1\right)+2 V_{K S}^{\prime 2}\left(1+i \alpha_{1}^{\prime} Q_{1}^{\prime}\right)\right\} A_{1}^{\prime}+\right. \\
& \left.+\left\{V_{K T}^{\prime 2}\left(Q_{2}^{\prime 2}-1\right)+2 V_{K S}^{\prime 2}\left(1+i \alpha_{2}^{\prime} Q_{2}^{\prime}\right)\right\} A_{2}^{\prime}\right] \text {. }
\end{aligned}
$$

From (11b) and (11e) we find that only possible values of $C$ and $C^{\prime}$ are zeros. Hence there is no propagation of displacement $u_{2}$. Thus no $S H$-waves occur in this case.

Eliminating the constants $A_{1}, A_{2}, A_{1}^{\prime}, A_{2}^{\prime}$ from (11a)-(11d) we obtain the wave velocity equation finally in determinant form as

where

$$
\left|M_{i j}\right|=0, \quad(i, j=1,2,3,4)
$$

and

$$
\begin{aligned}
M_{1 K} & =\left[1-i \alpha_{K} Q_{K}\right], \quad M_{1 K+2}=-\left[1+i \alpha_{K}^{\prime} Q_{K}^{\prime}\right] ; \quad[\text { when } K=1,2] \\
M_{2 K} & =\left[\alpha_{K}+i Q_{K}\right], \quad M_{2 K+2}=-\left[\alpha_{K}^{\prime}-i Q_{K}^{\prime}\right] ; \\
M_{3 K} & =\rho \times\left(V_{K S}^{2} / \eta_{K}^{*}\right)\left[2 i Q_{K}+\left(1+Q_{K}^{2}\right) \alpha_{K}\right], \\
M_{3 K+2} & =-\rho^{\prime} \times\left(V_{K S}^{\prime 2} / \eta_{K}^{* \prime}\right)\left[-2 i Q_{K}^{\prime}+\left(1+Q_{K}^{\prime 2}\right) \alpha_{K}^{\prime}\right] ; \\
M_{4 K} & =\left(\rho / \eta_{K}^{*}\right)\left[V_{K T}^{2}\left(Q_{K}^{2}-1\right)+2 V_{K S}^{2}\left(1-i \alpha_{K} Q_{K}\right)\right], \\
M_{4 K+2} & =\left(-\rho^{\prime} / \eta_{K}^{* \prime}\right)\left[V_{K T}^{\prime 2}\left(Q_{K}^{\prime 2}-1\right)+2 V_{K S}^{\prime 2}\left(1+i \alpha_{K}^{\prime} Q_{K}^{\prime}\right)\right] ;
\end{aligned}
$$

$$
Q_{K}=\left(1-\zeta_{K}^{2} / \eta^{2}\right)^{\frac{1}{2}}, \quad Q_{K}^{\prime}=\left(1-\zeta_{K}^{\prime 2} / \eta^{2}\right)^{\frac{1}{2}}, \quad(\text { when } K=1,2) .
$$

From (12) we get the wave velocity of surface waves in the common boundary under consideration in the presence of gravity and viscosity where viscosity is of first order 
including strain rate and stress rate simultaneously. Although, effects of viscosity and gravity are small, the present analysis should prove to be useful in circumstances where these influences cannot be neglected.

\section{Particular cases}

\subsection{Rayleigh waves}

To start with a particular case of surface waves, we consider visco-elastic Rayleigh waves under the influence of gravity in which the plane boundary is a free surface such that $M_{2}$ is replaced by vacuum. Here we also note that there can be no $S H$-waves.

Hence in view of (11d) and (11f), we obtain

$$
\begin{aligned}
{\left[2 i Q_{1}+\left(1+Q_{1}^{2}\right) \alpha_{1}\right] A_{1} } & +\left[2 i Q_{2}+\left(1+Q_{2}^{2}\right) \alpha_{2}\right] A_{2}=0 \\
{\left[V_{K T}^{2}\left(Q_{1}^{2}-1\right)+2 V_{K S}^{2}\left(1-i \alpha_{1} Q_{1}\right)\right] A_{1}+} & \\
+ & {\left[V_{K T}^{2}\left(Q_{2}^{2}-1\right)+2 V_{K S}^{2}\left(1-i \alpha_{2} Q_{2}\right)\right] A_{2}=0 . }
\end{aligned}
$$

After elimination of $A_{1}$ and $A_{2}$ from (13) and (14), we have

where

$$
\left|M_{i j}^{\prime}\right|=0, \quad(i, j=1,2)
$$

$$
\left.\begin{array}{ll}
M_{1 m}^{\prime} & =\left[2 i Q_{m}+\left(1+Q_{m}^{2}\right) \alpha_{m}\right] ; \\
M_{2 m}^{\prime} & =\left[V_{K T}^{2}\left(Q_{m}^{2}-1\right)+2 V_{K S}^{2}\left(1-i \alpha_{m} Q_{m}\right)\right] .
\end{array} \quad \text { (when } m=1,2\right),
$$

Equation (15) describes Rayleigh waves in a visco-elastic solid medium of the Voigt (1887) type under the influence of gravity including strain rate and stress rate. In the absence of viscous and gravitational fields, this equation tallies with the corresponding classical result (Bullen 1965, pp. 252-265).

\subsection{Love waves}

For Love type surface waves on the surface of the earth, we assume only the non-zero component of displacement $u_{2}$ which is a function of $x_{1}, x_{3}$ and $t$. All other components of displacement are zero. Let us assume that medium $M_{2}$ is obtained by two horizontal plane surfaces at a finite distance $H$ apart, the upper plane surface being free while the lower plane surface forms the medium $M_{1}$ and extends to an infinitely great distance.

For medium $M_{2}$ we must retain the full solution, since displacement no longer diminishes with increasing distance from the boundary surface of the two media and for medium $M_{1}$, solutions are the same as it is in the general case.

Therefore, for medium $M_{1}$, we write

$$
\begin{aligned}
u_{2}^{\prime}=\left[C_{1}^{\prime}\right. & \exp \left[x_{3}\right]\left(\eta^{2}-\omega^{2} \eta_{K}^{*^{\prime}} / V_{K S}^{\prime 2}\right)^{\frac{1}{2}}+ \\
& \left.+C_{2}^{\prime} \exp \left[-x_{3}\right]\left(\eta^{2}-\omega^{2} \eta_{K}^{*^{\prime}} / V_{K S}^{\prime 2}\right)^{\frac{5}{2}}\right] \exp \left[i\left(\eta x_{1}-\omega t\right)\right],
\end{aligned}
$$

where the restriction that the real part of $\left(\eta^{2}-\omega^{2} \eta_{K}^{* \prime} / V_{K S}^{\prime 2}\right)^{\frac{1}{2}}$ be positive is not required for $M_{2}$. 
In the present case boundary conditions are

(i) $u_{2}$ and $\sigma_{32}$ are continuous at $x_{3}=0$

(ii) $\sigma_{32}^{\prime}=0$, at $x_{3}=-H$.

Employing boundary conditions (i) and (ii) and using (9c) and (16), we get the following equations

$$
\begin{aligned}
& C=C_{1}^{\prime}+C_{2}^{\prime} \text {, } \\
& -\rho \times\left(V_{K S}^{2} / \eta_{K}^{*}\right)\left(\eta^{2}-\omega^{2} \eta_{K}^{*} / V_{K S}^{2}\right)^{t} \times C=\rho^{\prime} \times\left(V_{K S}^{\prime 2} / \eta_{K}^{*}\right) \times \\
& \times\left(\eta^{2}-\omega^{2} \eta_{K}^{* \prime} / V_{K S}^{\prime 2}\right)^{\frac{1}{2}}\left[C_{1}^{\prime}-C_{2}^{\prime}\right], \\
& C_{1}^{\prime} \exp \left[-H\left(\eta^{2}-\omega^{2} \eta_{K}^{*} / V_{K S}^{\prime 2}\right)^{\frac{1}{2}}\right]-C_{2}^{\prime} \exp \left[H\left(\eta^{2}-\omega^{2} \eta_{K}^{*} / V_{K S}^{\prime 2}\right)^{\frac{1}{2}}\right]=0 .
\end{aligned}
$$

Eliminating $C, C_{1}^{\prime}$ and $C_{2}^{\prime}$ from (17), (18) and (19), we get

$$
\begin{aligned}
\rho & \times\left(V_{K S}^{2} / \eta_{K}^{*}\right)\left(1-c^{2} \eta_{K}^{*} / V_{K S}^{2}\right)^{\frac{1}{2}}+\rho^{\prime} \times\left(V_{K S}^{\prime 2} / \eta_{K}^{*^{\prime}}\right)\left(\left(c^{2} \eta_{K}^{*^{\prime}} / V_{K S}^{\prime 2}\right)-1\right)^{\frac{1}{2}} \times \\
& \times \tan \left\{\eta H\left(\left(c^{2} \eta_{K}^{* \prime} / V_{K S}^{\prime 2}\right)-1\right)^{\frac{1}{2}}\right\}=0
\end{aligned}
$$

(where $c=\omega / \eta$ ), which is the required wave velocity equation for Love waves under the influence of gravity in a visco-elastic solid medium of first order including strain and stress rates. It is seen from (20) that Love waves do not depend upon the gravity field, although they depend upon the viscous field.

Taking $\eta_{0}=1$ and $\eta_{1}=\eta_{1}^{\prime}=\lambda_{1}=\lambda_{1}^{\prime}=\mu_{1}=\mu_{1}^{\prime}=0,(20)$ reduces to the corresponding result (Bullen 1965, pp. 252-265) in perfectly elastic medium.

\subsection{Stoneley waves}

The generalized forms of Rayleigh waves are Stoneley waves in which we assume that waves are propagated in the vicinity of interface of two semi-infinite media $M_{1}$ and $M_{2}$. Wave velocity of Stoneley waves in the presence of viscosity and gravity effects is determined by the roots of the equation (12) in the case of a visco-elastic medium of first order including strain and stress rates simultaneously. This equation of course reduces once more to the classical result in the absence of these effects.

\section{Discussion and conclusions}

It is clear from the above investigation that visco-elastic surface waves are affected by the time rate of strain and stress parameters. These parameters influence the wave velocity to an extent depending on the corresponding constants characterizing the visco-elasticity of the material. Further, the effect of gravity is significant when the wavelength is large, while the effect is small when the wavelength is small. However, there is always dispersion of waves due to gravity.

It is noted that Love waves are not affected by the gravity field in any way as is evident from the equations of motion. As regards Rayleigh waves in visco-elastic solid medium of the Voigt type under the influence of gravity, we find that the wave velocity equation proves that there is dispersion of waves in presence of gravity and viscosity. 
The results are in agreement with corresponding classical results when gravity and viscosity are neglected. This wave velocity equation is useful for numerical work.

The Stoneley wave velocity equation is very similar to the corresponding problem in the classical theory of elasticity. Here also there is dispersion of waves due to the presence of the gravity field and visco-elastic nature of the solid. This generalized type of surface wave in visco-elastic infinite solids accounting for gravity reduces to the classical Stoneley wave when gravity is absent.

\section{References}

Biot M A 1965 Mechanics of incremental deformation (New York: Wiley)

Bland D R 1960 The theory of linear visco-elasticity (London: Pergamon) (Monograph on the subject contains many cases of stress analysis)

Bromwich T J I A 1898 On the influence of gravity on elastic waves and, in particular on the vibrations of an elastic globe. Proc. London Math. Soc. 30: 98-120

Bullen K E 1965 An introduction to the theory of seismology (London: Cambridge University Press)

Das T K, Sengupta P R 1990a Surface waves in general visco-elastic media of higher order. Indian J. Pure Appl. Math. 21(7): 661-675

Das T K, Sengupta P R 1990b Surface waves in thermo-visco-elastic media considering time rate of stress and strain of higher order. Gerlands Beitr. Geophys. Leipzig 99: 337-448

De S N, Sengupta P R 1973 Plane Lamb's problem under the influence of gravity. Gerlands Beitr. Geophys. Leipzig 82: 421-426

De S N, Sengupta P R 1974 Influence of gravity on wave propagation in an elastic layer. J. Acoust. Soc. Am. 55: 919-921

Eringin A C, Suhubi E S 1975 Linear theory in elasto-dynamics (New York, London: Academic Press) vol. 2

Ewing W M, Jardetzky W S, Press F 1957 Elastic waves in layered media (London: McGraw-Hill)

Flugge W 1967 Visco-elasticity (London: Blaisdell)

Hunter S C 1960 Visco-elastic waves. In Progress in solid mechanics (eds) I N Sneddon, R Hill (Amsterdam, New York: North Interscience)

Jeffreys H 1959 The earth 4th edn (Cambridge: University Press)

Love A E H 1911 Some problems of geodynamics (New York: Dover)

Rayleigh Lord 1885 On waves propagated along the plane surface of an elastic solid. Proc. London Math. Soc. 17: 4-11

Roy S K, Sengupta P R 1983a Rotatory vibration of a sphere of general visco-elastic solid. Gerlands Beitr. Geophys., Leipzig 92: 70-76

Roy S K, Sengupta P R 1983b Radial vibration of a sphere of general visco-elastic solid. Gerlands Beitr. Geophys., Leipzig 92: 435-442

Stoneley R 1924 Elastic waves at the surface of separation of two solids. Proc. R. Soc., London 106: 416-428

Voigt W 1987 Theoretische studien über die Elasticitäts verhältnisse der Krystalle. Abh. Ges. Wiss. Göttingen 34 